\title{
Evaluación del nicho ambiental de Lycalopex fulvipes (zorro de Darwin) y la incidencia del cambio climático sobre su distribución geográfica
}

\section{Evaluation of the environmental niche of Lycalopex fulvipes (Darwin's fox) and the incidence of climate change on its geographic distribution}

\author{
Camila Molina, Andrea Castillo \& Horacio Samaniego*
}

Laboratorio de Ecoinformática. Instituto de Conservación, Biodiversidad y Territorio, Universidad Austral de Chile, Campus Isla Teja s/n, Los Ríos, Valdivia, Chile.

*E-mail: horacio@ecoinformatica.cl

\begin{abstract}
RESUMEN
Se modeló la distribución del zorro de Darwin (L. fulvipes) entre las regiones del Bío-Bío y Aysén bajo dos escenarios de clima futuro (RCP2.6 y RCP8.5). El modelo más optimista (RCP2.6) predice un incremento en el rango de distribución, contrario al modelo RCP8.5, que muestra extinciones locales, pero ganancias de hábitat en áreas donde no se ha registrado esta especie. Los resultados son evaluados respecto al sistema de áreas de protección existentes para esta especie endémica y amenazada del cono sur de Sudamérica.
\end{abstract}

Palabras Clave: Modelos de distribución de especies, nicho ambiental, cambio climático.

\begin{abstract}
The distribution of the Darwin fox (L. fulvipes) between the Bío-Bío and Aysén regions in Chile was modeled under two future climate scenarios (RCP2.6 and RCP8.5). The most optimistic RCP2.6 model predicts an increase in the range of distribution, contrary to the RCP8.5 model, which shows local extinctions, but habitats gains in areas where this species has not been registered. The results are evaluated with respect to the system of protected areas for this endemic and endangered species in the southern cone of South America.
\end{abstract}

KEYwords: Models of distribution species, environmental niche, climate change.

\section{INTRODUCCIÓN}

El antropoceno se ha caracterizado por las profundas alteraciones en los ecosistemas naturales atribuibles al cambio de uso del suelo y el cambio climático inducido por el hombre (Armesto et al. 2010, Lenoir et al. 2008; Franklin 2009; IPCC 2013). La evidencia apunta a que este acelerado cambio en los regímenes de temperatura y precipitación impactan seriamente a la biodiversidad (Araújo \& Rahbeck 2006, Pecl et al. 2017), incrementando las tasas de extinción locales, particularmente a aquellas especies con distribución geográfica restringida y limitada capacidad de dispersión (Thomas et al. 2004). Entre estas últimas, las especies endémicas poseen una menor capacidad de adaptación a un clima que cambia rápidamente, en comparación con otras especies (Ohlemüller et al. 2008). Es por ello, que las estrategias para la conservación de dichas especies deben ser capaces de anticipar los impactos del antropoceno y consecuentes cambios en el clima (Araújo \& Rahbek 2006). Los modelos de distribución de especies (MDE) se han convertido en una valiosa herramienta tanto para el estudio de los parámetros que definen el nicho ambiental de especies (Soberón et al. 2017), así como para comprender e identificar la distribución geográfica esperada dado un set de condiciones ambientales definidas a priori (Guisan \& Zimmermann 2000). Su simplicidad conceptual y práctica ha hecho los MDE particularmente populares (Hu \& Jiang 2011), sobre todo para la planificación de la conservación (Franklin 2009). En términos prácticos, la construcción y análisis de estos MDE posibilitan el estudio de los efectos antropogénicos sobre la biodiversidad y patrones asociados (Guisan \& Thuiller 2005). Esto, a través de la categorización 
de la importancia que poseen aquellas variables esenciales para la persistencia de las especies. El desarrollo de estos modelos, permiten por otro lado, describir el nicho ecológico de especies, y obtener una representación cartográfica de su rango de distribución geográfico potencial (Soberón et al. 2017). En este proceso es posible, además, utilizar dichos modelos para evaluar aquel set de variables que definen la distribución potencial actual y bajo escenarios climáticos futuros, u otro agente de cambio potencial a escala geográfica, como son los cambios de uso del suelo producto de la acción antrópica (Franklin 2009).

El zorro de Darwin o zorro chilote (Lycalopex fulvipes, Martin, 1837) es la más pequeña de las tres especies de cánidos que habitan en Chile y uno de los más pequeños del mundo (Iriarte \& Jaksic 2012). Con apenas 52 a $67 \mathrm{~cm}$ de longitud y un peso que varía entre 1,8 y $4 \mathrm{~kg}$ (Iriarte 2008), este zorro endémico del bosque templado lluvioso parece hoy restringido a Chile, y es catalogado como una de las especies con mayor prioridad de conservación a nivel mundial (Jiménez et al. 2008), siendo considerado En Peligro (C1) por la UICN (Silva-Rodríguez et al. 2016). Esto se debe a su restringido rango de distribución, tamaño poblacional reducido, y por estar expuesto a importantes amenazas en gran parte de su hábitat original (Cofré \& Marquet 1999; UICN 2012).

La literatura indica que se encuentra en al menos dos poblaciones distintas (Jiménez \& McMahon 2004) ubicadas en los bosques de la Isla de Chiloé, y en la cordillera costera continental; aunque recientes hallazgos sugieren que esta última población podría ser discontinua (Silva-Rodríguez et al. 2016). Hasta hace unos años, se creía que esta especie se encontraba restringida a la zona continental de la Cordillera de Nahuelbuta (Jiménez \& McMahon 2004). Sin embargo, estudios recientes han confirmado la presencia de la especie en la Cordillera de la Costa Valdiviana (D'Elía et al. 2013; Farías et al. 2014; Silva-Rodríguez et al. 2018) y en las cercanías de la desembocadura del río Maullín (SilvaRodríguez et al. 2016).

Dado el actual estado de conservación de este cánido endémico de Chile, y considerando los nuevos antecedentes en la distribución de sus poblaciones, este estudio busca presentar un análisis espacial basado en un MDE. Mediante la evaluación de aquellas variables climáticas que componen su nicho actual y proyectado, generamos mapas de distribución potencial actual y futuro según recientes predicciones de escenarios de cambio climático futuros. Con ello se espera, $a$ ) identificar aquellos factores climáticos que describen la distribución de esta especie, $b$ ) determinar aquellas áreas más favorables para la ocurrencia actual y futura del zorro chilote, y c) evaluar el efecto del cambio climático sobre la disponibilidad de hábitat futuro para esta especie. Los resultados de este estudio aportan nuevos antecedentes para apoyar la planificación y gestión de áreas para la conservación del zorro de Darwin.

\section{MATERIALES Y MÉTODOS}

DATOS DE OCURRENCIA

Los datos de ocurrencia de L. fulvipes fueron recopilados mediante la revisión exhaustiva de la bibliografía publicada en libros, revistas y consultas a GBIF con información georeferenciada. Se incluyeron, además, registros no publicados (i.e. cámara-trampa y heces). Se compiló un total de 169 puntos de ocurrencia colectados entre 1986 y 2017. En la región de La Araucanía, para Nahuelbuta, los trabajos revisados fueron el de Jiménez et al. (1991) con registro de visita en estación olfativa, Jiménez (2000) con ejemplares capturados y Sánchez et al. (2013) con registros de cámaras trampa; en Lastarria, Gorbea, se ocupó el trabajo de D'Elía et al. (2013) con un individuo muerto. Para la región de Los Ríos, en Punta Chachán se ocupó la publicación de Vilá et al. (2004) con un registro de piel (identificado genéticamente). El mismo trabajo se ocupó para el avistamiento directo del Fundo Chaihuín; en los registros del Parque Oncol, Parque Nacional Alerce Costero y Reserva Costera Valdiviana se utilizó la publicación de Farías et al. (2014), todos avistamientos por cámaras trampa (también Silva-Rodríguez et al. 2018). Para la zona continental de región de Los Lagos, en Puerto Octay se ocupó una observación personal de J. Jiménez incluida en Silva-Rodríguez et al. (2016) con avistamientos directos y por cámaras trampa; el registro de cerca de Maullín es un avistamiento personal con cámaratrampa de A. Farías, también incluido en Silva-Rodríguez et al. (2016). Para la Isla de Chiloé, en Piruquinca se utilizaron visitas en estación olfativas incluidas en Jiménez et al. (1991); en Pidpid y en Alcaldeo de Llau-llao, se utilizaron registros de heces del trabajo de Medel et al. (1990); en Ahuenco la ocurrencia se georreferenció con QGIS 2.14 en base a una imagen de las locaciones estimadas a partir de heces y animales capturados del trabajo de Jiménez (2007); en el Parque Nacional Chiloé los registros fueron por cámaras trampa y se encuentran en el trabajo de Azorín et al. (2014). Los datos obtenidos en GBIF corresponden a tres ocurrencias reportadas en la Isla de Chiloé, asociadas a las localidades Coinco, Púlpito y los lagos Huillinco y Tarahuín (iNaturalist.org 2018). Respecto a los datos no publicados, estos representan en su mayoría registros de cámara-trampa, esfuerzo de muestreo de A. Farías y del trabajo doctoral de Paula Zucolillo. Las ocurrencias abarcan el Parque Oncol, Parque Nacional Alerce Costero, y en la isla de Chiloé, específicamente Chepu, Caulín, Parque Nacional Chiloé, y los parques privados Ahuenco, Tepuhueico, y Tantauco. Este último fue muestreado en los sectores Yandal, Chaiguata y la ribera en la desembocadura Bahía Nayahua.

Área DE ESTUdio

La extensión del MDE se sitúa en la zona sur de Chile, entre los $-35^{\circ}$ y $-47^{\circ} \mathrm{S}$, y $\operatorname{los}-76^{\circ}$ y $-68^{\circ} \mathrm{W}$, abarcando de norte a sur las regiones administrativas Bío-Bío, La Araucanía, 
Los Ríos, Los Lagos y Aysén (MMA 2016). Su rango extiende desde la cordillera de Nahuelbuta $\left(37^{\circ} 11^{\prime} \mathrm{S}\right)$ por $175 \mathrm{~km}$ de norte a sur sobre la Cordillera de la Costa y una altura máxima de 1.350 m.s.n.m. (Smith-Ramírez 2004). La literatura indica que en Nahuelbuta, el zorro de Darwin habita en un ecosistema ecotonal, donde los bosques deciduos y matorrales mediterráneos convergen con la vegetación siempreverde valdiviana (Owen 2007). Su presencia se asocia a bosques densos (Jaksic et al. 1990; Jiménez et al. 1991), con mayor frecuencia de Araucaria-Nothofagus, bosques abiertos de Nothofagus y ocasionalmente en pastos abiertos (Silva-Rodríguez et al. 2016). Según Moreira-Arce et al. (2015), esta especie se asocia positivamente con la disponibilidad de bosques nativos, y negativamente con usos de suelo antropizados como carreteras y ambientes urbanos o peri-urbanos.

Los individuos avistados en la cordillera costera de la región de Los Ríos se asocian principalmente a bosques nativos viejos y secundarios, de hoja perenne de la selva valdiviana, aunque es posible situarlo ocasionalmente en plantaciones de Eucalyptus spp (Farías et al. 2014). La fisonomía de la región ha cambiado debido al uso agrícola y ganadero que ha primado en los últimos años (BCN 2016).

En Chiloé esta especie se asocia a la cadena montañosa, una prolongación de la Cordillera de la Costa (Segarra et al. 1990), con vegetación higromórfica en bosques siempreverdes (Farías \& Jaksic 2011; INE 2006). Se encontró que los zorros chilotes utilizan más bien bosques y matorrales de segundo crecimiento y en menor medida bosques antiguos. El uso de dunas es variable según el consumo de crustáceos (Elgueta et al. 2007; Jiménez 2007). Su ámbito de hogar varía entre 103 y 488 ha (Jiménez 2007). Por último, los registros cercanos al lago Llanquihue, en su distribución norte, muestran un bosque muy fragmentado, con granjas lecheras intensivas, de grandes pasturas y franjas lineales de bosque valdiviano degradado a lo largo de los arroyos (Obs. pers. J. Jiménez).

VARIABLES BIOCLIMÁTICAS ACTUALES Y PROYECCIONES FUTURAS Para modelar la distribución de L. fulvipes, se utilizaron las 19 variables bioclimáticas del proyecto WorldClim versión 1.4 (Hijmans et al. 2005). Este conjunto de datos resume información de precipitación y temperatura a partir de la interpolación de valores climatológicos de estaciones meteorológicas a nivel global a una resolución espacial de 1 $\mathrm{km}^{2}$. Un análisis de correlación por pares permitió disminuir la multicolinealidad, removiendo aquellas variables con alta correlación ( $\mathrm{r}>0,8)$ (Menard 2002; Guisan et al. 2017), disminuyendo así el sobreajuste del modelo (Beumont et al. 2005). Posteriormente, se comprobó la independencia del conjunto de variables finales seleccionadas con un análisis de componentes principales (ver Apéndice). Las variables seleccionadas fueron temperatura promedio anual (Bio1); isotermalidad (Bio3); temperatura máxima del mes más cálido (Bio5); precipitación anual (Bio12); precipitación del mes más húmedo (Bio13); y precipitación estacionaria (Bio15).

Para proyectar la distribución de esta especie a escenarios de cambio climático, se consideró la metodología propuesta por el Intergovernmental Panel on Climate Change (IPCC) utilizada en el Coupled Model Intercomparation Project 5 (CMIP5) para la generación del $5^{\text {to }}$ informe (AR5) (Taylor et al. 2012). Entre los 19 Modelos de Circulación Global o Global Circulation Models (GCM) del CMIP5, se seleccionó el modelo HadGEM2-ES o "HE" del Met Office Hadley Centre, U.K (Collins et al. 2011). Su versión anterior, el modelo HadCM3, ha sido utilizado para elaborar el Plan Nacional de adaptación al cambio climático de Chile (Universidad de Chile 2012; MMA 2014), así como también para modelar la distribución de otros cánidos sudamericanos como el aguará guazú (Queriolo et al. 2013), y a grupos de especies de rango pequeño en general (Ohlemüller et al. 2008). El modelo proyectado fue evaluado al mediano plazo (i.e. año 2070), bajo dos de los escenarios RCPs (Representative Concentration Pathways), 2.6 y 8.5. Estos escenarios representan concentraciones futuras de Gases de Efecto Invernadero (GEI), asociadas a los diferentes factores conocidos que afectan el cambio climático, como son la población mundial, el desarrollo económico, los sistemas de energía, el cambio de uso de la tierra, entre otros (Moss et al. 2010). El RCP2.6, es el escenario más optimista pues proyecta concentraciones futuras de GEI más bajas. Según este escenario, estás tendrán un valor máximo alrededor del año 2020 y producirán un aumento de la temperatura media global de $0,3^{\circ} \mathrm{C}$. En contraste, el segundo escenario RP8.5 representa el escenario más pesimista pues proyecta un crecimiento sostenido de los GEI hasta finales del siglo con un consecuente aumento de la temperatura media global de $2,6^{\circ} \mathrm{C}$ a $4,8^{\circ} \mathrm{C}$ (IPCC, 2013). En este trabajo se han seleccionado estos escenarios extremos de tal manera de poder analizar la probabilidad del impacto máximo y mínimo del calentamiento global sobre la distribución del zorro chilote. Por otro lado, estos mismos escenarios han sido utilizados para proyectar el cambio climático en Chile (Universidad de Chile 2012; DMC 2013; MMA 2014), así como para modelar la distribución futura de diferentes ecosistemas y taxas (Zuliani et al. 2015; Scriven et al. 2015; Jyväsjärvi et al. 2015; Pouteau et al. 2016).

\section{Modelación}

Se utilizó el principio de máxima entropía implementado en el software MaxEnt (versión 3.3.3k) para modelar la distribución actual y futura de L. fulvipes (Phillips et al.2006). Este software permite utilizar una base a datos de presencia y pseudo-ausencias junto a un set de variables ambientales para encontrar aquellas variables que mejor describen el nicho potencial de la especie analizada, estableciendo la idoneidad del hábitat o probabilidad potencial de encontrar 
a la especie en el área geográfica estudiada (Phillips et al. 2006; Franklin, 2009). MaxEnt es considerado hoy entre las mejores aproximaciones para el desarrollo de MDE, pues ha demostrado un buen desempeño en comparación con otras técnicas de modelación (Elith et al. 2006), tanto para estimar distribuciones actuales como para las distribuciones futuras bajo escenarios de cambio climático (Hijmans \& Graham, 2006). Además, Hernández et al. (2006) y Peterson et al. (2007) apoyan el uso de MaxEnt para modelar con tamaños de muestras pequeñas como las de este estudio. Es por estas razones que hoy en día es el método más comúnmente utilizado en la construcción de MDE (Merow et al. 2013).

Dada la baja cantidad de datos, se llevaron a cabo 30 réplicas de validación cruzada con las configuraciones predeterminadas para el parámetro de regularización $(\beta$ $=1$ ), el número máximo de iteraciones se ajustó a 1.000, el umbral de convergencia a $10^{-5}$ y el número máximo de puntos de fondo a 10.000 (Phillips et al. 2004). Estos parámetros han demostrado tener buen rendimiento en diversos grupos taxonómicos (Phillips \& Dudik 2008, Merow et al. 2013, Fourcade et al. 2014). Los datos de presencia se dividieron aleatoriamente en dos subconjuntos, uno de entrenamiento del modelo con $75 \%$ de los datos, y otro de prueba con los 25\% restantes (Phillips et al. 2006). Se utilizó la salida logística cuyos resultados son más fáciles de interpretar (Phillips \& Dudik 2008). En esta, se otorga a cada pixel una probabilidad de ocurrencia, o idoneidad del hábitat, donde $\mathrm{p}=0$ representa una probabilidad nula de ocurrencia de la especie, y $\mathrm{p}=1$ la probabilidad completa. Por último, se utilizó la opción fade by clamping para minimizar sobrestimaciones y evitar que las variables tomen valores fuera del rango del modelo de entrenamiento al proyectarse bajo escenarios de cambio climático (Phillips et al. 2006).

\section{EVALUACIÓN DEL MODELO Y PREDICCIONES ESPACIALES}

La confiabilidad de los modelos generados fue evaluada con un análisis umbral-independiente del área bajo la curva (AUC) proporcionado por el Receiver Operator Curve (ROC) (Fielding \& Bell 1997, Phillips et al. 2006; Acevedo et al. 2010; Anderson \& Raza 2010). Los valores van comúnmente de 0 a 1 . Se acepta la predicción de los modelos cuando se obtienen valores sobre 0,7 , y se considera que un modelo tiene un alto rendimiento cuando los valores exceden 0,9 (Fielding \& Bell 1997; Franklin, 2009). Un análisis Jackknife del AUC permitió conocer la contribución de cada variable a la generación del modelo de tal manera a identificar aquellas variables que mejor predicen la ocurrencia de la especie (Phillips et al. 2006). Finalmente, la proyección cartográfica del nicho actual y futuro del zorro de Darwin utilizó el modelo promedio de las 30 réplica con un umbral de idoneidad para el hábitat predicho de 0,25. La superficie de distribución de idoneidad resultante fue clasificada según Shrestha \& Bawa (2014), en tres clases de idoneidad: baja (25\%-50\% de la probabilidad de ocurrencia); media $(50 \%-75 \%$ de la probabilidad de ocurrencia); y alta ( $>75 \%$ de probabilidad de ocurrencia). Se consideró como hábitat no adecuado los valores bajo $25 \%$.

REDISTRIBUCIÓN DEL NICHO Y SU CONSERVACIÓN

Se cuantificó la superficie de distribución actual y futura del zorro de Darwin sobre la categorización de idoneidad (ver párrafo anterior). Se calculó, además, el cambio de superficie geográfica entre el modelo actual y los proyectados con un Sistema de Información Geográfico. Al traslapar las predicciones geográficas de cada modelo, se determinó el hábitat perdido (superficie de distribución que sólo se observa en el modelo actual pero que desaparece del modelo futuro); el hábitat ganado (superficie de distribución que sólo se observa en el modelo proyectado, no así en el modelo actual); y el hábitat conservado (superficie de distribución conservada en ambos modelos).

Finalmente se comparó la superficie del modelo actual y los modelos proyectados, con la superficie de las áreas protegidas (AP) Chilenas. Los datos de AP provienen del Catálogo Nacional de Información Geoespacial de Chile (IDE 2016). Se consideraron aquellas unidades de conservación categorizadas como Parques Nacionales, Reservas Nacionales, Monumentos Nacionales, Bienes Nacionales, Reservas de la Biósfera, sitios Ramsar (2012) y Áreas Protegidas Privadas (2011).

\section{RESULTADOS}

DeSEMPEÑO DE LOS MODELOS DE DISTRIBUCIÓN PARA EL ZORRO DE DARWIN

En los análisis de sensibilidad, el modelo actual y los modelos proyectados mostraron excelente desempeño medido por el alto valor promedio del $\operatorname{AUC}(>0,9)$.

Respecto a la contribución de las variables utilizadas en la generación de los modelos (Tabla 1), la mayor contribución corresponde a la precipitación anual. El valor de esta variable aumenta en los modelos futuros, y en mayor medida bajo el escenario más pesimista (i.e. RCP8.5). La isotermalidad, la precipitación estacionaria y la temperatura máxima del mes más cálido poseen una contribución similar en todos los modelos, mientras que la temperatura promedio anual y la precipitación del mes más húmedo, son menos significativas para los modelos con contribuciones menores al 1\%.

Los resultados del análisis Jackknife (Fig. 1) muestran que todas las variables contribuyen en diferentes porcentajes a la explicación del modelo con altos valores de AUC (> 0,81 ). Es decir, en todos los modelos, actual y proyectados, el conjunto de variables seleccionadas se desempeña de forma óptima y son igualmente importantes para explicar la distribución de los datos en el espacio geográfico analizado. 


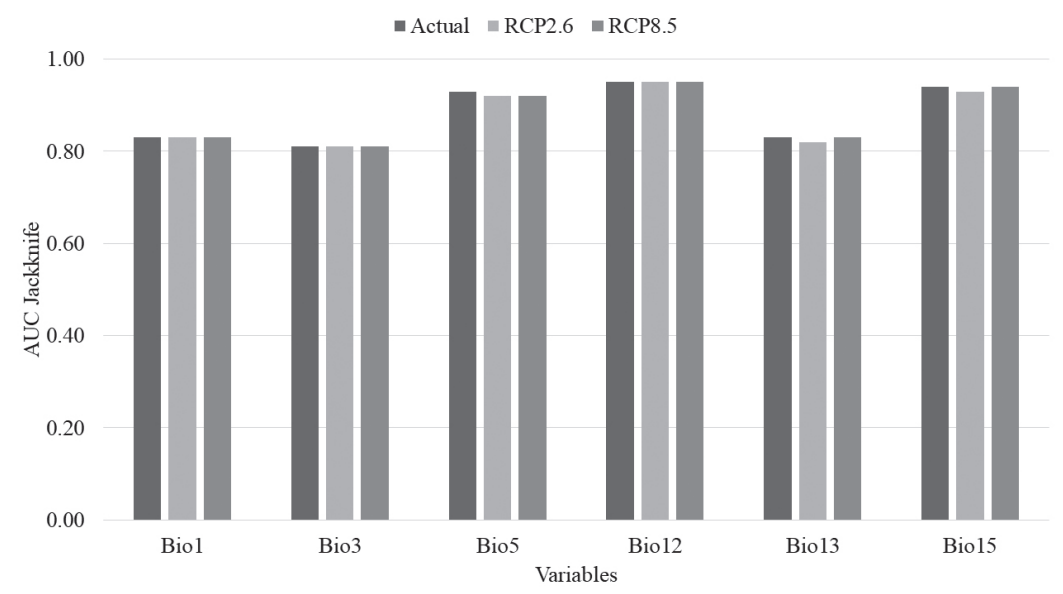

FigURA 1. Análisis Jackknife del AUC para variables bioclimáticas utilizadas en la generación de los modelos de distribución de especies en condiciones de clima actual y proyectado para L. fulvipes bajo escenarios RCP2.6 y RCP8.5. / Jackknife analysis of AUC for bioclimatic variables used in current and projected species distribution models of L. fulvipes under RCP2.6 and RCP8.5 scenarios of climate change.

TABLA 1. Porcentaje de la contribución relativa de las variables climáticas que componen el modelo actual y los modelos proyectados para L. fulvipes. *valores de la variable con mayor contribución. / Relative contribution percentajes of the climate variables used in current projected species distribution models for L. fulvipes. * indicates the variable with the largest contribution.

\begin{tabular}{lccc}
\hline VAriables & Modelo aCtUAL & Modelo RCP2.6 & Modelo RCP8.5 \\
\hline Temperatura promedio anual (Bio1) & 0,6 & 0,8 & 0,9 \\
Isotermalidad (Bio3) & 10,7 & 11,3 & 9,9 \\
Temperatura máxima del mes más cálido (Bio5) & 14,9 & 12,8 & 13,1 \\
Precipitación anual (Bio12) & $54,1 *$ & $56,3^{*}$ & $57,4^{*}$ \\
Precipitación del mes más húmedo (Bio13) & 0,5 & 0,4 & 0,2 \\
Precipitación estacionaria (Bio15) & 19,1 & 18,5 & 18,5 \\
\hline
\end{tabular}

\section{SUPERFICIE DE DISTRIBUCIÓN DE LOS MODELOS}

Las predicciones de las probabilidades de ocurrencia actual y proyectadas del zorro de Darwin, se observan en la Figura 2. Los resultados muestran que el modelo actual de esta especie proyecta una superficie de distribución potencial de $14.249 \mathrm{~km}^{2}$ (Fig. 2a). Por su parte, el modelo futuro proyectado al escenario RCP2.6 predice un aumento en la superficie de distribución del $32 \%$ con $18.752 \mathrm{~km}^{2}$ (Fig. 2b), mientras que el modelo proyectado al RCP8.5, pese a ser la proyección de cambio climático más pesimista, predice un incremento de casi 16\% del área de distribución respecto a la situación actual, con $16.503 \mathrm{~km}^{2}$ de hábitat calificado como idóneo (Fig. 2c).

En el modelo actual, L. fulvipes muestra una mayor probabilidad de ocurrencia $(>75 \%)$ en el NO de la Isla de Chiloé, y una media a baja probabilidad en el resto de Chiloé, al igual que en la costa de la región de Los Lagos y Los Ríos. No se observan áreas de idoneidad de hábitat $>25 \%$ en la Cordillera de Nahuelbuta en la región de La Araucanía. Bajo el escenario RCP2.6, estas áreas se expanden e indican un cambio en la disponibilidad de hábitat altamente idóneo para la especie en el sector costero de la región de los Ríos y el norte de la Isla de Chiloé. Se observa, además, alta idoneidad en el Archipiélago de las Guaitecas, en la región de Aysén, junto con la aparición de hábitat de baja calidad en el Cordillera de Nahuelbuta. Bajo el escenario RCP8.5, estas mismas áreas de distribución disminuyen con respecto a lo proyectado en el escenario RCP.26, con una pérdida total en la zona de Nahuelbuta y una disminución notoria en la costa de la región de los Ríos. La superficie predicha en la Isla Grande de Chiloé sufre pérdidas menores con una disminución en la calidad de sus hábitats, mientras que la probabilidad de ocurrencia del zorro chilote aumenta hacia el sur, en el Archipielago de las Guaitecas.

Para estimar el impacto del cambio climático en la distribución de L. fulvipes se cuantificó la superficie según categorías de idoneidad de hábitat para cada modelo construido. Los resultados se observan en la Figura 3. Bajo el escenario RCP2.6 se prevé un aumento significativo en las superficies de alta y media idoneidad de hábitat en un $488 \%$ y $24 \%$ para cada categoría respecto a la situación actual. El mayor incremento se registra en la superficie de alta idoneidad, el cual supera casi cinco veces la superficie de distribución actual de la especie. Bajo el escenario RCP8.5, el más pesimista, ocurre lo opuesto. Las superficies de alta y media idoneidad de hábitat disminuyen en un 57\% y $37 \%$ 
respectivamente, respecto de la situación actual. Mientras que la superficie de baja idoneidad incrementa en un $78 \%$. Esto significa que, bajo el escenario de cambio climático más severo, si bien la superficie total disponible para la distribución geográfica del zorro de Darwin aumentará en un $16 \%$, sólo prevalecerá la disponibilidad de hábitat de menor calidad (idoneidad).

La cuantificación del cambio de superficie total entre el modelo actual y los proyectados se exponen en la Tabla 2. Los análisis muestran que bajo el escenario RCP2.6, se conserva una superficie de $13.859 \mathrm{~km}^{2}$, se pierden $387 \mathrm{~km}^{2}$ respecto a la distribución actual, y se gana una superficie de $4.863 \mathrm{~km}^{2}$. Bajo el escenario RCP8.5, la superficie que se conserva es sólo de $9.755 \mathrm{~km}^{2}$, se pierde una superficie de $4.490 \mathrm{~km}^{2}$ respecto al modelo de distribución actual, y se gana una superficie de $6.712 \mathrm{~km}^{2}$. Los resultados muestran que en ambos escenarios la generación de nuevo hábitat supera las pérdidas en la superficie. Sin embargo,bajo el escenario más severo (RCP8.5) estos cambios parecen más abruptos.

TABLA 2. Cuantificación del cambio de superficie total entre el modelo actual y los modelos proyectados a escenarios de cambio climático futuros, RCP 2.6 y RCP 8.5. / Total surface change between current projected species distribution models under the mildest and more worst future climate change scenarios, RCP 2.6 and RCP 8.5 .

\begin{tabular}{lccc}
\hline \multirow{2}{*}{ Modelos comparados } & \multicolumn{3}{c}{ Superficie $\left(\mathrm{km}^{2}\right)$} \\
\cline { 2 - 4 } & Hábitat perdido & Hábitat conservado & Hábitat ganado \\
\hline Actual - RCP2.6 & 387 & 13.859 & 4.863 \\
Actual - RCP8.5 & 4.490 & 9.755 & 6.713 \\
\hline
\end{tabular}

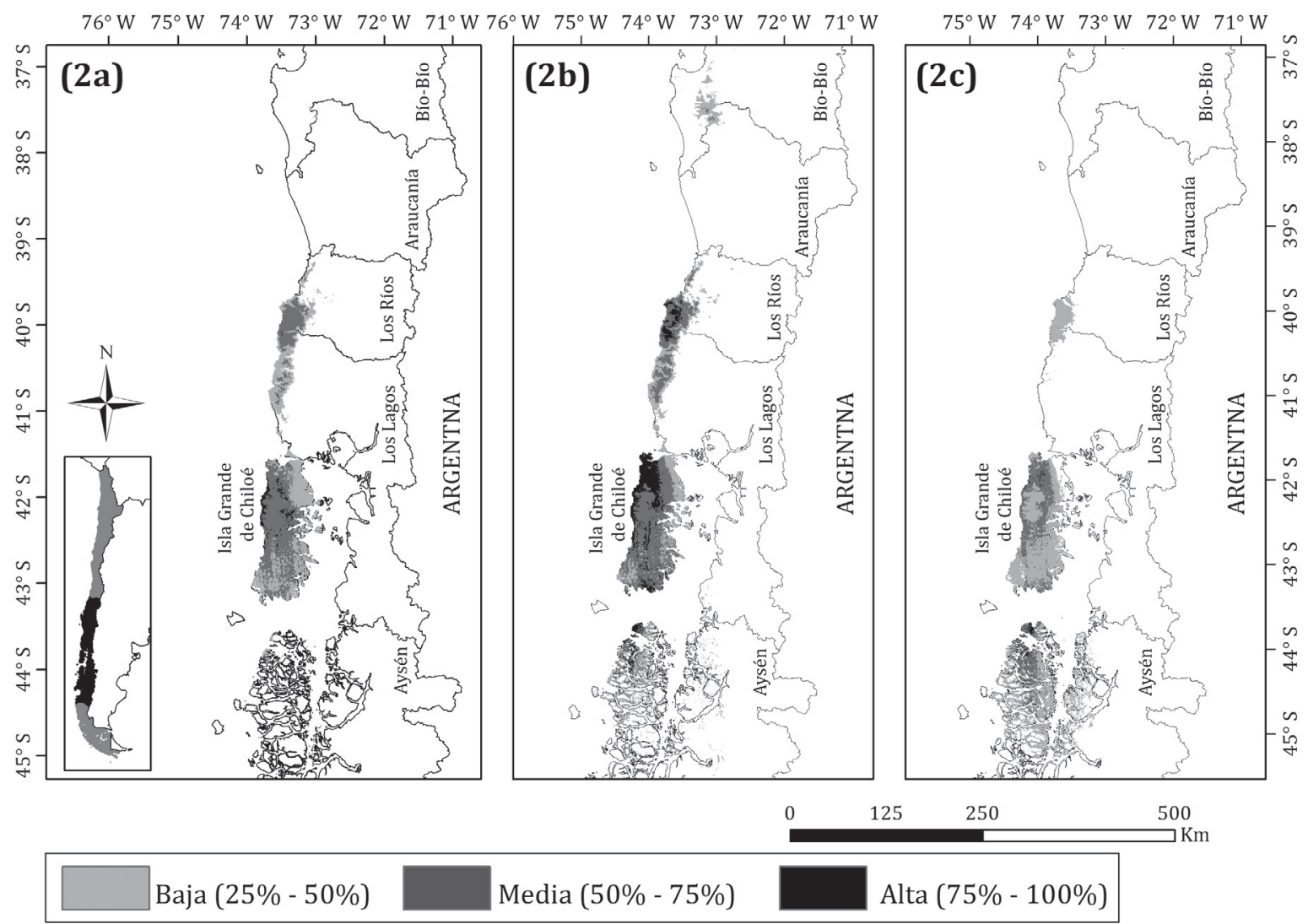

FIgURA 2. Predicción espacial de la probabilidad de ocurrencia de L. fulvipes. El panel (2a) corresponde al modelo actual; el panel (2b) al modelo bajo el escenario RCP2.6; y el panel (2c) al modelo bajo el escenario RCP8.5. La probabilidad de ocurrencia (o idoneidad del hábitat) fue categorizada en baja (25\%-50\%) representada en color gris claro, la probabilidad de ocurrencia media (50\%-75\%) en color gris oscuro, y la alta probabilidad de ocurrencia (>75\%) en color negro. / Spatial distribution of L. fulvipes occurrence probability. Panel (2a) corresponds to current distribution model; panel (2b) to distribution modeled under the RCP2.6 scenario; and panel (2c) to distribution modeled under the RCP8.5 scenario. The probability of occurrence (or habitat suitability) was categorized as low (25\% $-50 \%)$ in light gray, medium probability $(50 \%-75 \%)$ in dark gray, and high probability $(>75 \%)$ in black. 


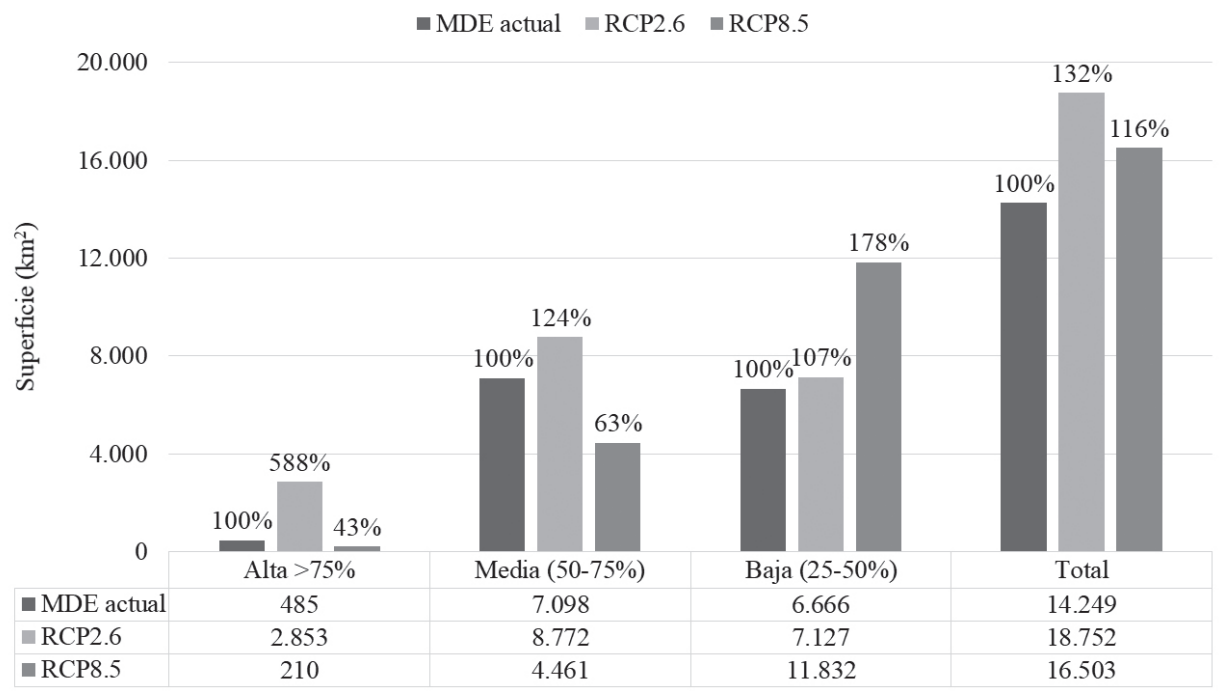

Figura 3. Superficie en $\mathrm{km}^{2}$ de cada modelo según categoría de idoneidad de hábitat. Los valores sobre las columnas corresponden al porcentaje de ganancia o pérdida respecto a la superficie predicha por el modelo actual en cada categoría. $/ \mathrm{Area}^{\text {in }} \mathrm{km}^{2}$ of each model according to habitat suitability category. Values in the bottom table are percentages of gain (or loss) with respect to the area predicted by the current model in each category.
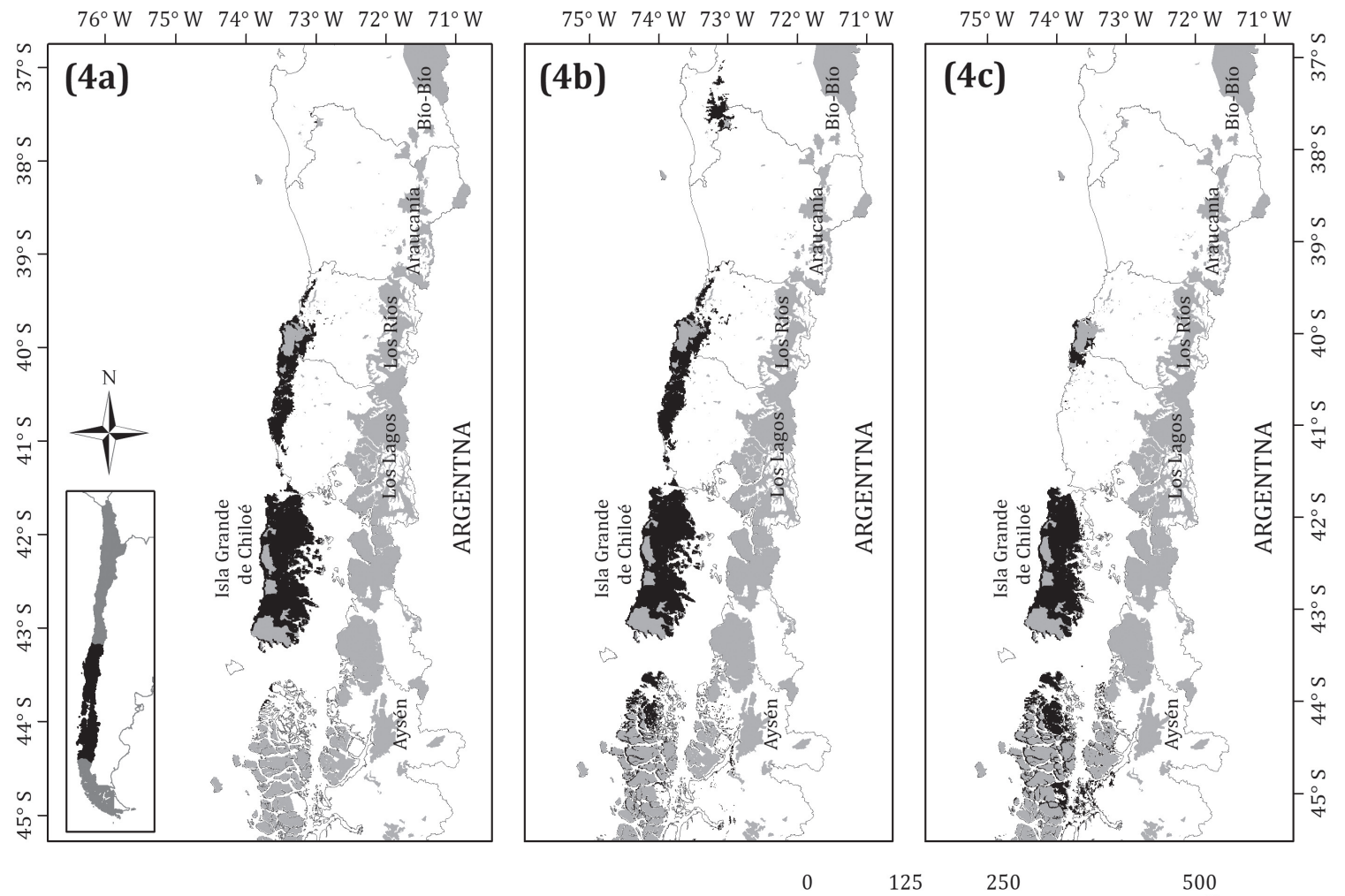

L. fulvipes (distribución > 25\%) Áreas protegidas

Figura 4. Superposición de los modelos de distribución actual y proyectados de L. fulvipes (superficie negra) con la distribución de áreas de protección en Chile (superficie gris). El panel (4a) corresponde al modelo actual, el panel (4b) al modelo bajo el escenario RCP2.6, y el panel (4c) al modelo bajo el escenario RCP8.5. / Overlap of current and projected distribution patterns of L. fulvipes (black surface) and protected areas in Chile (gray surface). Panel (4a) corresponds to current model, panel (4b) to model under the RCP2.6 scenario, and panel (4c) to model under the RCP8.5 scenario. 
DISTRIBUCIÓN DEL ZORRO DE DARWIN EN ÁREAS DE CONSERVACIÓN Las Áreas protegidas (AP) comprendidas entre las regiones del Bío-Bío y Aysén abarcan una superficie de 85.203 km², la que se sobrepone sólo en un 3,2\% (2.696 km²) del rango de distribución actual de la especie (Fig. 4a). Contrario a lo esperado, esta situación varía a favor del zorro chilote al proyectar su nicho a escenarios de cambio climático.

Bajo el escenario RCP2.6, la superficie actual de las AP sólo cubrirá un 4,6\% (3.928 $\left.\mathrm{km}^{2}\right)$ de la distribución futura del zorro de Darwin (Fig. 4b). Mientras que este porcentaje aumenta a 6,1\% $\left(5.159 \mathrm{~km}^{2}\right)$ bajo el escenario RCP8.5 (Fig. $4 c)$. Si bien la variación entre la actual superficie protegida y la proyectada no es significativa en términos estadísticos, es importante destacar que sólo una pequeña fracción de las AP a nivel nacional incluyen hábitat susceptible de ser ocupado por el zorro chilote, estas son: Los Parques Nacionales Nahuelbuta, Alerce Costero y Chiloé; en la región de Los Ríos el predio Llancahue (Bien Nacional Protegido) y el sitio Ramsar en el río Cruces, y AP Privadas entre las que se destacan el Parque Oncol (propiedad de Forestal Arauco S.A.), la Reserva Costera Valdiviana (The Nature Conservancy), el predio San Martín (Universidad Austral de Chile); y los Senderos de Chepu, junto a los Parques Tepuhueico, Ahuenco, Tantauco en la Isla de Chiloé. De la misma manera, al examinar los modelos proyectados se incluyen: el Parque Nacional Isla Magdalena, la Reserva Nacional las Guaitecas y la Reserva Natural Melimoyu en la región de Aysén.

\section{DISCUSIÓN}

Durante los últimos años, los MDEs se han impuesto como una herramienta aceptada por la comunidad científica para comprender los requerimientos de las especies para adaptarse y persistir en el ambiente que las rodea (Franklin 2009; Peterson et al. 2011). Como parte de este ejercicio, los MDE no sólo permiten definir el nicho ambiental, sino que también permiten proyectar los potenciales efectos del calentamiento global sobre la distribución de la biota (Lawing \& Polly 2011). Es un hecho de que las especies ejercen su potencial de adaptación al clima desplazando su rango de distribución a nuevas localizaciones, quedando muchas veces confinadas a microrefugios (Lenoir et al. 2008; Lenoir et al. 2017). La creciente evidencia científica sugiere que estos cambios de distribución no sólo afectan a aquellas dinámicas propias de la biota, como es el ciclaje de elementos esenciales para el funcionamiento de los ecosistemas como el nitrógeno y otros elementos; sino a las propias dinámicas asociadas al clima mismo, algo sin precedentes desde el último máximo glacial (Pecl et al. 2017). Aunque actualmente existen esfuerzos por limitar la emisión de GEI para contener el calentamiento a no más de $2^{\circ} \mathrm{C}$ (en el mejor de los escenarios), se espera que la redistribución de las especies afectará a gran parte de la biota debido a la enorme inercia presente en el sistema global de circulación climático y oceánico (IPCC 2013; Pecl et al. 2017). Entre las especies inmediatamente afectadas por el cambio climático, se encuentran las especies endémicas y especialistas de hábitat, las cuales merecen particular atención (Ohlemüller et al. 2008). Así, este trabajo es, a nuestro entender, la primera aproximación a nivel regional que estudia el nicho ambiental de L. fulvipes y los potenciales efectos que el cambio climático tendrá sobre su distribución geográfica.

\section{MODELO DE NICHO AMBIENTAL Y PREDICCIONES FUTURAS}

Las estructuras de los modelos obtenidos son coherentes con la historia natural y la autoecología del zorro de Darwin. De hecho, el MDE actual descrito aquí, tiene una importante similitud, al menos cualitativa, a la descrita en recientes atlas de distribución para la especie (Iriarte 2018). A partir del análisis aquí expuesto, es posible relacionar el alto porcentaje de contribución de la precipitación anual $(>54 \%)$ con la productividad vegetal en los tipos de ecosistemas en los que esta especie habita, y con los roles ecológicos que desempeña como depredador de micromamíferos y dispersor de semillas (Iriarte \& Jacksic 2012). L. fulvipes habita preferentemente bosques densos, de vegetación hidrófita, con sotobosque bien formado, y de condiciones de alta humedad que favorece el mesofitismo (INE 2007), los cuales propician una mayor abundancia de frutos y pequeños vertebrados que son parte de la dieta principal de esta especie (Iriarte \& Jaksic 2012). Entre estos últimos destaca el ratón colilarga, Oligoryzomys longicaudata (Elgueta et al. 2007), principal vector de virus hanta vector de una enfermedad con alta mortalidad en seres humanos.

Los modelos aquí propuestos predicen tres zonas disjuntas dónde se distribuye actualmente el zorro de Darwin; (i) la Cordillera de Nahuelbuta, (ii) la costa de la región de Los Ríos y parte de la costa norte de Los Lagos y (iii) la Isla Grande de Chiloé. Estos resultados son congruentes con la existencia de poblaciones aisladas de esta especie, incluyendo las recientemente descritas en la región de Los Ríos (Farías et al. 2014). La falta de datos de muestreo entre estas zonas puede ser la razón de estos resultados. Por otra parte, creemos que la actual distribución del zorro de Darwin se debe a su ocurrencia en microrefugios climáticos. En estas áreas geográficas las condiciones ambientales han sido relativamente estables respecto a los pasados y actuales cambios climáticos (hipótesis de climas relictos) y han protegido a la especie de eventos de extinción (Ohlemüller et al. 2008; Lenoir et al. 2017). Un ejemplo de ello es la retracción y expansión de los glaciares durante el Pleistoceno y el Holoceno temprano, donde la cordillera de la Costa en la zona centro-sur de Chile corresponde al límite del Último Máximo Glacial (Clapperton 1983). Durante este periodo ocurre la diversificación de los cánidos en Sudamérica (Perini et al. 2010), donde L. fulvipes presenta una temprana 
radiación en el género Lycalopex spp. (Fuentes-González \& Muñoz-Durán, 2012). Creemos el género Lycalopex spp. pudo presentar una dinámica de especiación similar al de los roedores en Chile, del tipo "valle", en dónde las poblaciones se aíslan durante los periodos de glaciación, pero reanudan el intercambio genético y aumentan su dispersión durante los periodos interglaciales (contrario al caso del género Liolaemus spp) (Fuentes \& Jaksic 1979). Esto podría haber limitado la radiación del género, pero, además, dando como resultado una distribución discontinua con poblaciones aisladas en el caso de L. fulvipes.

Debe considerarse además que la fuerte reducción de la superficie histórica de bosque valdiviano, sin duda, ha tenido un rol más reciente en la discontinuidad de la distribución observada. Resulta evidente de todas las contribuciones revisadas para este análisis, que más allá de las condiciones climáticas que definen su nicho, las poblaciones de zorro chilote parecen concentrarse en los grandes remanentes de bosque nativo, por lo que resulta difícil distinguir la contribución relativa propuesta entre la hipótesis expuesta en párrafo precedente con el simple efecto de la deforestación más allá de las condiciones climáticas que definen el nicho de la especie. Así, resultaría particularmente interesante saber si realmente existe una asociación con las variables climáticas identificadas en este análisis, o si la correlación resultantes se deriva del hecho de tratarse de las condiciones climáticas en las áreas de acceso más difícil y por lo tanto menos impactadas por la deforestación.

Respecto a las proyecciones futuras, el modelo más optimista de cambio climático (RCP2.6) es sumamente favorable para el zorro chilote. Nuestro análisis indica que bajo este escenario se generará más hábitat nuevo del que se pierde respecto al modelo actual ( $+34 \%$ de superficie) Lo mismo ocurre con el modelo más pesimista, donde el desplazamiento del nicho hacia la región de Aysén hace que la producción de hábitat nuevo $(+47 \%)$ supere la superficie perdida en su área norte de distribución. Sin embargo, se debe tener muy presente dos cosas; por un lado, el escenario RCP2.6 es considerado el menos realista de los propuestos por la IPCC, ya que supone cambios rápidos y severos en las políticas sociales y ambientales, difíciles de llevar a cabo durante esta década (DMC 2013; IPCC 2013). Por otro lado, bajo el escenario RCP8.5, si bien la superficie de distribución aumenta respecto al modelo actual, nuestro análisis sugiere que la superficie de hábitat ganada será de media a baja idoneidad, e incluye la expansión de la distribución del zorro chilote hacia el Archipiélago de las Guaitecas, un área donde no existe indicio, ni registro alguno de su presencia a la fecha. Se observa, además, una severa disminución del hábitat en la Cordillera de Nahuelbuta donde encontramos hoy la población más pequeña de zorro de Darwin. Esto convierte al escenario RCP8.5 en la proyección menos favorable (pero más probable) para esta especie.
CAMBio CLIMÁtico Y ÁREAS DE CONSERVACIÓN

En Chile, los estudios de cambio climático que se han desarrollado a la fecha, señalan que la zona sur del país sufrirá un aumento en las temperaturas en torno a los $0,5^{\circ} \mathrm{y}$ $1{ }^{\circ} \mathrm{C}$, y una disminución de las precipitaciones entre un 5\% a $15 \%$ bajo los modelos RCP2.6 y RCP 8.5 respectivamente (DCM 2013; MMA 2014). En este sentido, este trabajo es un primer acercamiento al impacto de estos cambios en el clima sobre la distribución del zorro de Darwin y la identificación de aquellas áreas que deben ser destinadas a la conservación de esta especie.

Se debe considerar que, si bien es esperable que las especies respondan al cambio climático modificando sus rangos de distribución, o adaptándose a las nuevas condiciones de su entorno (Pecl et al. 2017), su incapacidad para dispersarse o adaptarse a condiciones locales ha de favorecer la formación de focos aislados o "refugios" donde esta especie experimente una mayor susceptibilidad a la extinción (Lenoir et al. 2017; Thomas et al. 2004; VeraEscalona et al. 2012). Los análisis comparativos entre las Áreas Protegidas y los modelos de distribución del zorro muestran baja coincidencia entre las áreas protegidas y su distribución actual $(3,2 \%)$ y las proyecciones aquí predicen un interesante incremento sobre este porcentaje (i.e. $4,6 \%$ y el $6,1 \%$ respectivamente).

Aunque estos resultados revelan que pese al cambio climático la distribución del zorro chilote se desplazará hacia regiones con mayor presencia de áreas protegidas, estos porcentajes siguen siendo muy bajos respecto de las cifras propuestas como porcentajes mínimos de representatividad en áreas protegidas (Leadley et al. 2010). Hoy en día, la red de APs con potencial de protección para el zorro de Darwin está principalmente asociada al PN Nahuelbuta en la zona norte junto a algunas áreas protegidas privadas con poca superficie que sólo podrían albergar poblaciones pequeñas de L. fulvipes. En el centro de su rango de distribución, en la región de Los Ríos y de Los Lagos, se aprecia claramente el aporte de privados, particularmente el Parque Oncol y la Reserva Costera Valdiviana. Esta última de mayor extensión es donde se ha fotografiado recientemente a la especie (Silva-Rodríguez et al. 2018). Por su parte en la región de Los Lagos, el esfuerzo de conservación de la Red de Parques Indígenas Mapu Lahual, podrían asegurar la conectividad a lo largo de la Cordillera de la Costa entre estas regiones. Por último, en Chiloé se observan muchos parches desconectados que están en manos de propietarios privados y comunidades indígenas. Bajo esta situación es necesaria una mayor vinculación entre los parques estatales (Parques Chiloé), los parques privados Tepuhueico, Ahuenco y Tantauco (todos de grandes extensiones), y los pequeños propietarios, para así generar una conservación más integral que satisfaga de manera efectiva los requisitos ecológicos del zorro de Darwin. 
ACTUALES AMENAZAS Y DESAFíOS PARA CONSERVACIÓN DEL ZORRITO DE DARWIN

Actualmente, el zorro chilote se encuentra amenazado por diversos factores, entre ellos, los bajos números poblacionales, la pérdida de hábitat, y la depredación y transmisión de enfermedades por parte de perros domésticos y asilvestrados. Estas son las principales causas por las que L. fulvipes está categorizado como En Peligro (C1) por la UICN (Silva-Rodríguez et al. 2016), y están dadas en parte, por la matriz que rodea a la distribución de esta especie. Esta se encuentra compuesta principalmente por suelos productivos del tipo forestal, agrícola y ganaderos. Se presume que podrían actuar como barreras para la dispersión, sin embargo, el registro de individuos en plantaciones (Farías et al. 2014) y en cercanía a lugares habitados, ponen en tela de juicio los requerimientos de hábitat no perturbados de esta especie. Esto último puede deberse a una mayor capacidad adaptativa de esta especie a distintos usos del suelo, que, junto a su comportamiento altamente oportunista, le confiere cierta facilidad para sobrevivir en ambientes más heterogéneos que lo pensado, y con menor disponibilidad de presas (Iriarte \& Jaksic 2012). Sin embargo, una matriz heterogénea ha de incluir también carreteras y áreas urbanas que podrían ejercer mayor presión sobre el hábitat de esta especie, propiciando el ingreso de otras amenazas como el riesgo de atropello, caza ilegal, domesticación, etc. Es necesario ahondar más en el efecto de estas amenazas antrópicas, un ejemplo de ello es el estudio de Moreira-Arce et al. (2015).

La suma de todas estas amenazas y presiones tienen como consecuencia la reducción del tamaño efectivo de las poblaciones del zorro de Darwin, haciendo que esta especie sea más propensa a la extinción por eventos fortuitos (i.e. estocasticidad ambiental y poblacional). Hoy la pregunta más grande es qué hacer en las zonas en las que se prevé la presencia de zorros en un futuro, por ejemplo, en el Archipiélago de las Guaitecas. Es claro que si bien nuestro modelo muestra cierta idoneidad de hábitat en dicho archipiélago, son áreas aisladas donde difícilmente podrán trasladarse individuos sin asistencia, por lo que más allá de la aptitud climática, no resulta relevante considerar un aumento efectivo de la distribución de la especie debido a las limitaciones para la dispersión impuesta por la barrera marítima. Es por ello, que la tarea a corto plazo es fortalecer las áreas protegidas en las que potencialmente se encontrará a esta especie, con énfasis en mitigar las amenazas anteriormente descritas. Creemos que estos objetivos pueden lograrse a través de la planificación en conjunto con los actores públicos y privados, reforzando por sobre todo, el traspaso de información a los pequeños propietarios que ejercen conservación y turismo. A largo plazo, se espera que futuros planes de protección beneficien al zorro chilote, como podrían ser la creación de nuevas áreas protegidas en áreas de alta idoneidad, la creación de corredores biológicos o un centro de reproducción como ya se pensó en Chiloé en el 2015 (MINAGRI, 2015). Hoy en día, los cambios en la distribución de las especies producto del cambio climático, requerirán modificaciones en los objetivos de las leyes de conservación en general, que han tradicionalmente enfatizado la conservación in situ y la retención de las condiciones históricas. Estas necesitan considerar que las especies irán más allá de sus rangos geográficos históricos para adaptarse a condiciones favorables, y así evitar invertir en proteger lugares donde la especie ya no será viable (Pecl et al. 2017).

A la fecha, ya se han realizado diversas iniciativas en esta dirección para la conservación de este cánido, entre ellas se destaca el "Plan Nacional de Recuperación, Conservación y Gestión (RECOGE) del Zorro de Darwin" (2012) y el "Proyecto de Conservación del Zorro de Darwin" de la Fundación Buin Zoo (2013). Por otro lado, en Nahuelbuta se realizó un proyecto de conservación en la interface PN Nahuelbuta-áreas bordes, a cargo del Comité Iniciativa Nahuelbuta (2012-2014). Otros en relación a la implementación de medidas de mitigación de las principales amenazas, a cargo de la Universidad Católica de Temuco y CONAF (2013) y tres campañas de vacunación y desparasitación de perros y gatos en torno al PN Nahuelbuta a cargo del MMA y CONAF (2012, 2014-2015 y 2016). Por su parte, en Chiloé también se han se ha realizado proyectos, como el dirigido por el investigador Jaime Jiménez, el proyecto "Misión Zorro Chilote" y el aporte de la ONG Chiloé Silvestre. Se espera que estas iniciativas sean replicadas o retomadas por parte de las entidades a cargo.

Finalmente, se sugiere para próximos análisis, contar con más datos de ocurrencia, especialmente en aquellas zonas donde se ha avistado recientemente. Estimamos que para lograr proyecciones futuras más precisas, es necesario desarrollar modelos que consideren (i) la influencia de variables antrópicas como el cambio de uso de suelo, dado que la vegetación es un determinante clave de la presencia de esta especie (Moreira-Arce et al. 2015); y evaluar (ii) la disponibilidad de microrefugios climáticos, importantes para la protección de especies raras o endémicas que responden generalmente a este patrón de redistribución (Lenoir et al. 2017).

\section{AGRADECIMIENTOS}

Agradecemos a Eduardo Silva-Rodríguez por sus comentarios en los estadios tempranos de la preparación de este manuscrito, así como la valiosa contribución de Ariel Farías y Paula Zucalillo con preciosa evidencia geolocalizada de zorro de Darwin. Este trabajo contó con el financiamiento de CONICYT a través del proyecto FONDECYT \#1161280 a HS. Esta contribución es producto de un trabajo final del curso CBIT241 "Ecología de Paisaje" 
Nicho ambiental de Lycalopex fulvipes: Molina, C. ET AL.

impartido en la carrera de Ingeniería en Conservación de Recursos Naturales en la Universidad Austral de Chile.

\section{REFERENCIAS}

Araújo, M., RahbeK, C. 2006. How does climate change affect biodiversity? Science 313(5792): 1396-1397. DOI: 10.1126/science. 1131758

Armesto, J.J., Manuschevich, D., Mora A., Smith-Ramirez, C., Rozzi, R., Abarzúa, A.M., Marquet, P.A. 2010. From the Holocene to the Anthropocene: A historical framework for land cover change in southwestern South America in the past 15,000 years. Land Use Policy 27(2):148-160. DOI:10.1016/j.landusepol.2009.07.006

Acevedo, P., Ward, A.I., Real, R., Smith, G.C. 2010. Assessing biogeographical relationships of ecologically related species using favorability functions: A case study on British deer. Diversity and Distribution 16(4): 515-528. DOI:10.1111/j.1472-4642.2010.00662.x

Anderson, R.P., RAZA, A. 2010. The effect of the extent of the study region on GIS models of species geographic distributions and estimates of niche evolution: Preliminary tests with montane rodents (genus Nephelomys) in Venezuela. Journal of Biogeography 37(7):1378-1393. DOI:10.1111/ j.1365-2699.2010.02290.x

Azorín, B., Sillero, A., Delgado, F. 2014. Detección de huillín (Lontra provocax) y zorro chilote (Lycalopex fulvipes) en el Parque Nacional Chiloé. Biodiversidata CONAF (1):1718.

Biblioteca Del Congreso Nacional De Chile. 2016. Clima y vegetación región de los Ríos. URL:http://www.bcn.cl/ siit/nuestropais/region14/clima.html. Accedido: Abril 26, 2016.

Clapperton, C.M. 1983. The glaciation of the Andes. Quaternary Science Reviews 2(2-3): 85-155. DOI:10.1016/02773791(83)90005-7

Cofre, H., Marquet, P.A. 1999. Conservation status, rarity, and geographic priorities for conservation of Chilean mammals: an assessment. Biological Conservation 88(1): 53-68. DOI:10.1016/S0006-3207(98)00090-1

Collins, W.J., Bellouin, N., Doutriaux-Boucher, M., Gedney, N., Halloran, P., Hinton, T., Hughes, J., Jones, C.D., Joshi, M., Liddicoat, S., Martin, G., O'Connor, F., Rae, J., Senior, C., Sitch, S., Totterdell, I., Wiltshire, A., Woodward, S. 2011. Development and evaluation of an Earth-system model - HadGEM2. Geoscientific Model Development 4(4): 997-1062. DOI:10.5194/gmdd-4-9972011

DMC. 2013. Resumen: Análisis de los resultados Convenio Alta Dirección Pública, Director, Dirección Meteorológica de Chile, Objetivo $\mathrm{N}^{\circ} 3$. Dirección Meteorológica de Chile, Chile.

D’elía, G., Ortloff-Trautmann, A., Sánchez, P., Guiñez, B., VARAS, V. 2013. A new geographic record of the endangered Darwin's fox Lycalopex fulvipes (Carnivora: Canidae): Filling the distributional gap. Revista Chilena de Historia Natural 86(4): 485-488. DOI:10.4067/S0716078X20130004,00010
Elgueta, E.I., Valenzuela, J., Rau, J.R. 2007. New insights into the prey spectrum of Darwin's fox (Pseudalopex fulvipes Martin, 1837) on Chiloé Island, Chile. Mammalian Biology 72(3): 179-185. DOI: 10.1016/j.mambio.2006.07.004

Elith, J., Graham, C.H, Anderson, R.P, Dudík, M., Ferrier, S., Guisan, A., Hijmans, R.J., Huettmann, F., Leathwick, J.R., Lehmann, A., Li, J., Lohmann, L.G., Loiselle, B.A., Manion, G., Moritz, C., Nakamura, M., Nakazawa, Y., McC. Overton, J., Peterson, A.T., Phillips, S.J., Richardson, K., Scachetti-Pereira, R., Schapire, R.E., Soberón, J., Williams, S., Wisz, M.S., Zimmermann, N.E., AraúJo, M. 2006. Novel methods improve prediction of species' distributions from occurrence data. Ecography 29(2): 129-151. DOI:10.1111/j.2006.0906-7590.04596.x

Farías, A.A., Sepúlveda, M.A., Silva-Rodríguez, E.A., Eguren, A., González, D., Jordán, N.I., Ovando, E., Stowhas, P., Svensson, G.L. 2014. A new population of Darwin's fox (Lycalopex fulvipes) in the Valdivian Coastal Range. Revista Chilena de Historia Natural 87(1): 3. DOI:10.1186/0717-6317-1-3

FARÍAS, A.A., JAKSIC, F. M. 2011. Low functional richness and redundancy of a predator assemblage in native forest fragments of Chiloé Island, Chile. Journal of Animal Ecology 80(4): 809-817. DOI:10.1111/j.13652656.2011.01824.x

Fielding, J.H., Bell, A.F. 1997. A review of methods for the assessment of prediction errors in conservation presence/ absence models. Environmental Conservation 24(1): 3849. DOI: $10.1017 / \mathrm{S} 0376892997000088$

Fourcade, Y., Engler, J.O., RödDer, D., SECondi, J. 2014. Mapping species distributions with MAXENT using a geographically biased sample of presence data: A performance assessment of methods for correcting sampling bias. PLoS one 9(5): e97122. DOI:10.1371/journal.pone.0097122

Franklin, J. 2009. Mapping species distribution. Spatial inference and prediction. Ecology, Biodiversity and Conservation. Cambridge University Press, United Kingdom. DOI: 10.1017/CBO9780511810602

Fuentes, E.R., JAKSIC, F.M. 1979. Lizards and rodents: an explanation for their relative species diversity in Chile. Archivos de Biología y Medicina Experimental (Chile) 12: $179-190$.

Fuentes-González, J.A., Muñoz-Durán, J.M. 2012. Filogenia de los cánidos actuales (Carnivora: Canidae) mediante análisis de congruencia de caracteres bajo parsimonia. Actual Biology 34(96): 85-202.

Guisan, A., Thuiller, W., Zimmermann, N. E. 2017. Habitat suitability and distribution models: with applications in R. Cambridge University Press, United Kingdom. DOI: $10.1017 / 9781139028271$

Guisan, A., Thuiller, W. 2005 Predicting species distribution: offering more that simple habitat models. Ecology Letters 8(9): 993-1009. DOI:10.1111/j.1461-0248.2005.00792.x

Guisan, A., Zimmermann, N.E. 2000. Predictive habitat distribution models in ecology. Ecological Modelling 135(2-3): 147186. DOI:10.1016/S0304-3800(00)00354-9

Hernandez, P.A., Graham, C.H., Master, L.L., Albert, D.L. 2006. The effect of sample size and species characteristics on performance of different species distribution modeling methods. Ecography 29(5): 773-785. DOI:10.1111/j.0906- 
7590.2006.04700.x

Hijmans, R., Cameron, S., Parra, J., Jones, P., Jarvis, A. 2005. Very high resolution interpolated climate surfaces for global land areas. Journal of Climatology 25(15): 19651978. DOI: $10.1002 /$ joc. 1276

Hijmans, R.J., Graham, C.H. 2006. The ability of climate envelope models to predict the effect of climate change on species distributions. Global Change Biology 12(12): 2272-2281. DOI:10.1111/j.1365-2486.2006.01256.x

Hu, J., Jiang, Z. 2011. Climate change hastens the conservation urgency of an endangered ungulate. PloS one 6(8): e22873. DOI:10.1371/journal.pone.0022873.

INATURALIST. 2018. iNaturalist Research-grade Observations. iNaturalist.org. Occurrence Dataset. URL: https://doi. org/10.15468/ab3s5x accessed via GBIF.org. Accedido: Mayo 15, 2018.

Infraestructura De Datos Geoespaciales. 2016. Medio ambiente Archivos. URL: http://www.ide.cl/descarga/capas/category/ medio-ambiente.html. Accedido: Septiembre 20, 2016

INE. 2007. Región de la Araucanía, División política, administrativa y censal. Instituto Nacional De Estadísticas. URL: http:// www.inearaucania.cl. Accedido: Abril 10, 2016

INE. 2006. Chile: división Político-administrativa Censal 2001. Instituto Nacional De Estadísticas. URL: http://www. ine.cl/ine/canales/chile estadistico/territorio/division politico_administr ativa/pdf/dpa2001.pdf. Accedido: Abril 9, 2016.

IPCC. 2013. Climate Change, 2013. The Physical Science Basis. Contribution of Working Group I to the Fifth Assessment Report of the Intergovernmental Panel on Climate Change. Cambridge, United Kingdom and New York, NY, USA.

Iriarte, J.A. 2008. Mamíferos de Chile. Lynx Ediciones. Barcelona, España. 420 pp.

Iriarte, A., JAKSic, F.M. 2012. Lycalopex fulvipes. En: Los carnívoros de Chile: 148-153.

Jaksic, F.M., Jiménez, J.E., Medel, R.G., Marquet, P.A. 1990. Habitat and diet of Darwin's fox (Pseudalopex fulvipes) on the Chilean mainland. Journal of Mammalogy 71(2): 246-248. DOI: $10.2307 / 1382176$

JiMÉnEZ, J.E. 2000. Viability of the endangered Darwin's Fox (Pseudalopex fulvipes): Assesing ecological factors in the last mainland population and its ecology on the island population. Progress Report for the Lincoln Park Zoo Neotropic Fund.

JiMÉNEZ, J.E. 2007. Ecology of a coastal population of the critically endangered Darwin's fox (Pseudalopex fulvipes) on Chiloé Island, southern Chile. Journal of Zoology 271(1): 63-77. DOI:10.1111/j.1469-7998.2006.00218.x

Jiménez, J.E., Lucherini, M., Novaro, A.J. 2008. Pseudalopex fulvipes. IUCN red list of threatened species, Version 2012.2. URL: http://www.iucnredlist.org. Accedido: Marzo 14, 2017.

Jiménez J.E., Marquet P.A., Medel R.G., Jaksic F.M. 1991. Comparative ecology of Darwin's fox (Pseudalopex fulvipes) in mainland and island settings of southern Chile. Revista Chilena de Historia Natural 63: 177-186.

Jiménez, J.E., Mcmahon, E. 2004. Pseudalopex fulvipes (Martin, 1837) Critically Endangered-CR: C2a(ii) (2004) Darwin's fox. En: Canids: Foxes, Wolves, Jackals and Dogs.

Jyväsjärvi, J., Marttila, H., Rossi, P.M., Ala-Aho, P., Olofsson,
B., Nisell, J., Backman, B., Ilmonen, J., Virtanen, R., Paasivirta, L., Britschgi, R., Kløve, B., Muotka, T. 2015. Climate-induced warming impose a threat to north European spring ecosystems. Global Change Biology 21(12): 4561-4569. DOI:10.1111/gcb.13067

Lawing, A.M., Polly, P.D. 2011. Pleistocene climate, phylogeny, and climate envelope models: an integrative approach to better understand species' response to climate change. PLoS One 6(12): e28554. DOI:10.1371/journal. pone.0028554

Leadley, P.W., Krug, C.B., Alkemade, R., Pereira, H.M., Sumaila, U.R., Walpole, M., Marques, A., Newbold, T., T.E.H., L.S.L, van Kolck, J., Bellard, C., JanuchowskiHartley, S.R., Mumby, P.J. 2014. Progress towards the Aichi Biodiversity Targets: An Assessment of Biodiversity Trends, Policy Scenarios and Key Actions. Secretariat of the Convention on Biological Diversity, Montreal, Canada. Technical Series 78, 500 pp.

Lenoir, J., Gégout, J.C., Marquet, P.A., De Ruffray, P., Brisse, H. 2008. A significant upward shift in plant species optimum elevation during the $20^{\text {th }}$ century. Science 320(5884): 1768-1771. DOI: 10.1126/science.1156831.

Lenoir, J., Hattab, T., Pierre, G. 2017. Climatic microrefugia under anthropogenic climate change: implications for species redistribution. Ecography 40(2): 253-266. DOI: 10.1111/oik.02629.

Martin, W. 1837. Observations upon a new fox from Mr. Darwin's collection (Vulpes fulvipes). Proceedings of the Zoological Society of London 5: 11-12.

Medel, R.G., Jiménez, J.E., Jaksic, F.M., Yañez, J., Armesto, J.J. 1990. Discovery of a continental population of the rare Darwin's fox, Dusicyon fulvipes (Martin, 1837) in Chile. Biological Conservation 51(1):71-77. DOI:10.1016/00063207(90)90033-L.

Menard, S.W. 2002. Applied Logistic Regression Analysis. Sage Publications, Vol.106. Thousand Oaks, USA.

Merow, C., Smith, M.J., Silander, J.A. 2013. A practical guide to MaxEnt for modeling species' distributions: What it does, and why inputs and settings matter. Ecography 36(10): 1058-1069. DOI: 10.1111/j.1600-0587.2013.07872.x

MINAGRI. 2015. Trabajan para instalar centro de rehabilitación y conservación de la fauna silvestre en Chiloé. Ministerio De Agricultura. URL: http://oslagos.minagri. gob.cl/2015/01/15/trabajan-para-instalar-centro-derehabilitacion-y-conservacion-de-la-fauna-silvestre-enchiloe. Accedido: Mayo 5, 2017

MMA. 2016. Nuevos avistamientos del zorro de Darwin amplían su área de distribución. Ministerio Del Medio Ambiente. URL: http://portal.mma.gob.cl/nuevos-avistamientosdel-zorro-de-darwin-amplian-su-area-de-distribucion. Accedido: Abril 20, 2017.

MMA. 2014. Plan Nacional de adaptación al cambio climático. Ministerio Del Medio Ambiente. Santiago, Chile.

Moreira-Arce, D., Vergara, P.M., Boutin, S. 2015. Diurnal human activity and introduced species affect occurrence of carnivores in a human-dominated landscape. PLoS one 10(9): 1-19. DOI:10.1371/journal.pone.0137854

Moss, R.H., Edmonds, J.A., Hibbard, K.A., Manning, M.R., Rose, S.K., Van Vuuren, D.P., Carter, T.R., Seita Emori, S., Kainuma, M., Kram, T., Meehl, A.G., Mitchell, J.F.B., 
Nakicenovic, N., Riahi, K., Smith, S.J., Stouffer, R.J., Thomson, A.M., Weyant, J.P., Wilbanks, T.J. 2010. The next generation of scenarios for climate change research and assessment. Nature 463(7282): 747-56. DOI:10.1038/ nature 08823

Ohlemüller, R., Anderson, B.J., Araújo, M.B., Butchart, S.H.M., Kudrna, O., Ridgely, R.S., Thomas, C.D. 2008. The coincidence of climatic and species rarity: high risk to small-range species from climate change. Biology Letters 4(5): 568-572. DOI: $10.1098 / \mathrm{rsbl} .2008 .0097$

Owen, E. 2007. Informe final: Una evaluación rápida de la biodiversidad de dos predios integrados a la Red Conservacionista del Patrimonio Natural de Contulmo. En: Conservación de la biodiversidad de Nahuelbuta: Manejo sustentable y creación de áreas protegidas privadas en Contulmo. Chile, 60 pp.

Pecl, G.T., Araújo, M.B., Bell, J.D., Blanchard, J., Bonebrake, T.C., Chen, I.C., Clark, T.D., Colwell, R.K., Danielsen, F., Evengård, B., Falconi, L., Ferrier, S., Frusher, S., Garcia, R.A., Griffis, R.B., Hobday, A.J., JanionScheepers, C., Jarzyna, M.A., Jennings, S., Lenoir, J., Linnetved, H.I., Martin, V.Y., Mccormack P.C., Mcdonald, J., Mitchell, N.J., Mustonen, T., Pandolfi, J.M., Pettorelli, N., Popova, E., Robinson, S.A., Scheffers, B.R., Shaw, J.D., Sorte, C., Strugnell, J.M., Sunday, J.M., Tuanmu, M.N., Vergés, A., Villanueva, C., Wernberg, T., Wapstra, E., Williams, S.E. 2017. Biodiversity redistribution under climate change: Impacts on ecosystems and human well-being. Science 355(6332): eaai9214. DOI:10.1126/science.aai9214

Perini, F.A., Russo, C.A., Schrago, C.G. 2010. The evolution of South American endemic canids: A history of rapid diversification and morphological parallelism. Evolutionary Biology 23(2): 311-322. DOI: 10.1111/j.14209101.2009.01901.x

Peterson, R.G., Raxworthy, C.J., Nakumura, M., Peterson, A.T. 2007. Predicting species distribution from small numbers of occurrence records: a test case using cryptic geckos in Madagascar. Biogeography 34(1): 102-117. DOI: 10.1111/j.1365-2699.2006.01594.x

Peterson, A.T., Soberón, J., Pearson, R.G., Anderson, R.P., Martínez-Meyer, E., NaKamura, M., AraúJo, M.B. 2011. Ecological niches and geographic distributions. No. 49. Vol. 56, Princeton University Press, New Jersey, USA. DOI: 10.1644/1545-1542-94.1.241

PhILliPS, S.J., Dudík, M. 2008. Modeling of species distribution with Maxent: new extension and comprehensive evaluation. Ecography 31(2): 161-175. DOI: 10.1111/j.09067590.2008.5203.x

Phillips, S. J., Dudík, M., Schapire, R.E. 2004. A maximum entropy approach to species distribution modeling. In: Proceedings of the twenty-first international conference on Machine learning, pp. 83. DOI: 10.1145/1015330.1015412

Phillips, S., Anderson, R., Schapire, R. 2006. Maximun entropy modeling of species geographic distribution. Ecological Modelling 190(3-4): 231-259. DOI: 10.1016/j.ecolmodel.2005.03.026.

Pouteau, R., Meyer, J.Y., Blanchard, P., Nitta, J.H., Terorotua, M., TAPUtuarai, R. 2016. Fern species richness and abundance are indicators of climate change on high-elevation islands: evidence from an elevational gradient on Tahiti (French Polynesia). Climate Change 138(1-2): 143-156. DOI:10.1007/s10584-016-1734-x

Queirolo, D., Indrusiak, C., Coelho, L., Cosse, M., Dotta, G. 2013. Maned Wolf conservation in the South of Brazil and Uruguay. En: Consorte-McCrea, A.G., SAntos, E.F. 2013. Ecology and Conservation of the Maned Wolf: Multidisciplinary Perspectives. CRC Press, Boca Raton, USA, 344 páginas. ISBN: 9781466512603.

Sánchez, P., Guíñez, B., Cárcamo, J., Rojas, C. 2013. Conservación del zorro de Darwin (Lycalopex fulvipes) implementando medidas de mitigación a sus principales amenazas en el Parque Nacional Nahuelbuta, Región de La Araucanía. Biodiversidata CONAF, Boletín 2: 83-88.

Scriven, S.A., Hodgson, J.A., McClean, C.J., Hill, J.K. 2015. Protected areas in Borneo may fail to conserve tropical forest biodiversity under climate change. Biological Conservation 184: 414-423. DOI:10.1016/j.biocon.2015.02.018

Segarra, F., Rayo, G., Tosca, G. 1990. Situación actual y perspectivas del sector campesino en Chiloé. Consultorías profesionales agrarias, Santiago, Chile.

Silva-Rodríguez, E.A., Farías, A.A., Moreira-Arce, D., Cabello, J., Hidalgo-Hermoso, E., Lucherini, M., JimÉnez, J.E. 2016. Lycalopex fulvipes. The IUCN Red List of Threatened Species 2016: e.T41586A85370871.

Silva-Rodríguez, E.A., Ovando, E., González, D., Zambrano, B., Sepúlveda, M.A., Svensson, G.L., Cárdenas, R., Contreras, P., FARÍAs, A.A. 2018. Large-scale assessment of the presence of Darwin's fox across its newly discovered range. Mammalian Biology 92: 45-53. DOI: 10.1016/j. mambio.2018.04.003.

Shrestha, U.B., BawA, K.S. 2014. Impact of climate change on potential distribution of chinese caterpillar fungus (Ophiocordyceps sinensis) in Nepal Himalaya. PLoS one 9(9):e106405. DOI:10.1371/journal.pone.0106405

Smith-Ramírez, C. 2004. The Chilean Coastal Range: a vanishing center of biodiversity and endemism in South American temperate rainforests. Biodiversity and Conservation 13(2): 373-374. DOI: 10.1023/B:BIOC.0000006505.675 $60.9 \mathrm{f}$

Soberón, J., Osorio-Olvera, L., Peterson, T. 2017. Diferencias conceptuales entre modelos de nichos y modelación de áreas de distribución. Revista Mexicana de Biodiversidad 88(2): 437-441. DOI: 10.1016/j.rmb.2017.03.011

Taylor, K.E., Stouffer, R.J., Meehl, G.A. 2012. An overview of CMIP5 and the experiment design. Bulletin of the American Meteorological Society 93(4): 485-498. DOI: 10.1175/ BAMS-D-11-00094.1

Thomas, C.D., Cameron, A., Green, R.E., Bakkenes, M., Beaumont, L.J., Collingham, Y.C., Erasmus, B., Ferreira de Siqueira, M., Grainger, A., Hannah, L., Hughes, L., Huntly, B., van JaArsveld, A., Midgley, G., Miles, L., Ortega-Huerta, M.A., Peterson, A.T., Phillips, O., WilLIAMS, S. 2004. Extinction risk from climate change. Nature 427(6970): 145-148. DOI: 10.1038/nature02121

UICN. 2012. Lista roja de las especies amenazadas. Versión 2012.2 URL: http://www.iucnredlist.org. Accedido: Abril 15, 2016.

Universidad DE Chile. 2012. Estado del arte de modelos para la investigación del cambio global. Departamento de Geofí- 
sica, Facultad de Ciencias Físicas y Matemáticas, Universidad de Chile, Santiago, Chile.

Vera-Escalona, I., D’Elía, G., Gouin, N., Fontanella, F.M., Muñoz-Mendoza, C., Sites J.W., Victoriano, P.F. 2012. Lizards on ice: Evidence for multiple refugia in Liolaemus pictus (Liolaemidae) during the Last Glacial Maximum in the Southern Andean Beech Forests. PLoS one 7(11): e48358. DOI: 10.1371/journal.pone.0048358

Vila, C., Leonard, J., Iriarte, A., O’Brien, S., Johnson, W.,
WAYNE, R. 2004. Detecting the vanishing populations of the highly endangered Darwin's fox, Pseudalopex fulvipes. Animal Conservation forum 7(2): 147-153. DOI: 10.1017/ S1367943004001271

Zuliani, A., Massolo, A., Lysyk, T., Johnson, G., Marshall, S., Berger, K., Cork, S.C. 2015. Modelling the northward expansion of Culicoides sonorensis (Diptera: Ceratopogonidae) under future climate scenarios. PLoS one 10(8): e0130294. DOI: 10.1371/journal.pone.0130294

Recibido: 27.07 .2017

Aceptado: 28.05.2018 